\title{
Nocturnal evolution of respiratory effort in obstructive sleep apnoea syndrome: influence on arousal threshold
}

\author{
E. Sforza, J. Krieger, C. Petiau
}

Nocturnal evolution of respiratory effort in obstructive sleep apnoea syndrome: influence on arousal threshold. E. Sforza, J. Krieger, C. Petiau. (OERS Journals Ltd 1998.

ABSTRACT: It has been recently described that the overnight increase in maximal end-apnoeic oesophageal pressure (Poes,max), considered as an index of the arousal threshold to occlusion, mostly contributes to apnoea lengthening during the night. However, the rise in apnoea duration could also be caused by changes in hypoxaemia, chemosensitivity and upper airway resistance.

To better define the relative contributions of each of these factors, we examined the recordings of nine patients. Before apnoea, the mean pulmonary resistance at peak inspiratory flow (RPIF) was computed. During apnoea, all swings in oesophageal pressure $\left(P_{0}\right.$ es) were measured to define the $P_{\text {oes,max }}$, the increase from the minimum to the maximum $(\triangle P$ oes $)$, the rate of increase in Poes (RPoes) and the $P$ oes at the first occluded breath $(P$ oes, 1$)$.

A gradual and significant increase in apnoea duration $(p=0.02), P_{0 e s, \max }(\mathrm{p}=0.02)$ and $\Delta P_{0 \text { os }}(p=0.006)$ was present across the night without any changes in oxygen satu-

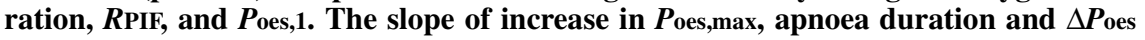
was correlated with the apnoea/hypopnoea index.

We conclude that in obstructive sleep apnoea, the nocturnal rise in apnoea duration is attributable more to an increase in the arousal threshold related to apnoea recurrence than to changes in chemosensitivity and upper airway resistance.

Eur Respir J 1998; 12: 1257-1263.
Sleep Disorders Unit, University Hospital, Strasbourg, France.

Correspondence: E. Sforza

Clinique Neurologique

F-67091 Strasbourg Cedex

France

Fax: 33388116343

Keywords: Arousal threshold nocturnal trend obstructive sleep apnoea oesophageal pressure

Received: December 121997 Accepted after revision July 71998
Resolution of obstructive apnoea during sleep is usually associated with a transient cortical arousal restoring upper airway patency and allowing resumption of ventilation. Hypoxaemia, hypercapnia, and increased respiratory effort $[1,2]$ may generate chemical and mechanical reflexes activating, directly or via central respiratory neurons, the reticular brainstem nervous system and allowing resumption of ventilation. Recent evidence in humans $[3,4]$ and in animals [5-7], has stressed that the progressive increase in the activity of respiratory muscles during an obstructive sleep apnoea (OSA) is the most important factor eliciting arousal and restoring upper airway patency. Thus, the maximum level of inspiratory effort (the maximal oesophageal pressure $\left(P_{\text {oes,max }}\right)$ ) preceding arousal has been considered as an index of the arousal threshold to occlusion.

In patients with OSA, apnoea frequency and length increase in the second part of the night $[8,9]$ associated with an increase in $P$ oes,max. This progressive rise in the index of the arousal threshold may occur throughout a night [10], as well as in the course of the disease [11]. Thus, we can hypothesize that the rise in apnoea length and in $P$ oes,max in OSA patients may be determined by changes in arousability related to the effects of sleep fragmentation and sleep deprivation [12]. However, the reported studies did not investigate any potential effects of sleep fragmentation, changes in upper airway collapsibility and respira-

For editorial comments see page 1239 tory drive during the night, and the analysis were limited to selected apnoeas during the first and last hours of the night [10].

The current study was designed to determine whether the recurrence of obstructive apnoeas was associated with a progressive rise in the index of arousal threshold and in apnoea length across the night. If so, we analysed which diurnal or nocturnal variables (including indices of chemosensitivity and preapnoeic pulmonary resistance) affect the nocturnal rise in respiratory effort and in apnoea duration.

\section{Patients and methods}

Nine male patients, diagnosed as having OSA and chosen from our database, were examined. The patients were eligible if they had: 1) a nocturnal polysomnography performed using pneumotachograph and oesophageal pressure recordings without any technical problems during the monitoring; 2) a diagnostic study in the supine position throughout the night with repetitive apnoeas during all 1-h periods; 3 ) at least $7 \mathrm{~h}$ of nocturnal recording with periods of awake state lasting <30 min in each 1-h period; and 4) at least 10 obstructive apnoeas per hour eligible for analysis.

None of the patients suffered from acute lung disease at the time of the study, and none had received previous treatment with nasal continuous positive airway pressure 
(CPAP) or surgery. All patients had a full evaluation including polysomnography, blood-gas analysis and respiratory function tests for diagnostic purposes. They were informed that some of the collected data would be used for research purposes, and they gave written informed consent.

Nocturnal polysomnography was carried out between 22:00-23:00 $\mathrm{h}$ and at 06.00 $\mathrm{h}$ using an electroencephalogram (EEG), electro-oculogram (EOG) and electromyogram (EMG) of chin muscles for conventional sleep staging. Breathing was analysed with a Fleisch \#2 pneumotachograph and electronic integrator (Godart Statham, Bilthoven, the Netherlands) attached to a face mask. Oxygen saturation $\left(\mathrm{Sa}, \mathrm{O}_{2}\right)$ was measured continuously with an earlobe oximeter (Biox III; Ohmeda, Boulder, CO, USA). Oesophageal pressure was measured with a $10-\mathrm{cm}$ latex balloon placed in the lower third of the oesophagus, inflated with $1 \mathrm{~mL}$ of air and connected to a pressure transducer (Validyne MW 45; Validyne, Northridge, CA, USA). The reference pressure for oesophageal pressure measurements was the atmospheric pressure and consequently all inspiratory pressures were negative. To facilitate analysis, all pressure values are given in absolute values, so that increases in the respiratory effort correspond to increases in the oesophageal pressure swings.

Sleep was scored using the criteria of RECHTSCHAFEN and KALES [13] for 20-s periods, and the following variables were calculated: total sleep time, wake time after sleep onset (WASO), sleep efficiency (total sleep time/total recording time $\times 100$ ), and the percentage of stages $1,2,3$, and 4 and rapid eye movement (REM) sleep. As indices of sleep fragmentation, we calculated the number of arousals, awakenings and sleep state changes. Arousal was defined as an EEG shift in the alpha or theta frequency having a duration of 3-10 s associated with an increase in chin EMG and awakening as a transition to at least one 20 -s period of wakefulness. Respiratory events were defined using standard criteria. Hypopnoeas were defined as a Š $50 \%$ reduction in tidal volume from the baseline value lasting at least $10 \mathrm{~s}$. The apnoea index (AI) and the apnoea + hypopnoea index (AHI) were established as the ratio of the number of apnoeas and apnoeas+hypopnoeas per hour of sleep. The mean $\mathrm{Sa}_{\mathrm{a}} \mathrm{O}_{2}$ during 10 min of quiet wakefulness prior to sleep onset, the minimal $\mathrm{Sa}_{\mathrm{a}} \mathrm{O}_{2}$ and the mean of the minimal $\mathrm{Sa}_{\mathrm{a}} \mathrm{O}_{2}$ after each event (mean lowest $\mathrm{Sa}_{\mathrm{a}} \mathrm{O}_{2}$ ) were also computed.

Conventional spirography was performed with a $10-\mathrm{L}$ closed spirograph, and static lung volumes were measured using the closed circuit helium dilution method.

\section{Data analysis}

Analyses were carried out for periods of $1 \mathrm{~h}$ from lightoff to light-on for $7 \mathrm{~h}$ during non-REM (NREM) sleep. Oesophageal pressure was measured for all obstructive apnoeas with the following rejection criteria: 1) apnoeas with artifacts in oesophageal pressure or oxygen saturation tracings; and 2) apnoeas occurring during the 15-min periods before and after REM sleep periods. Obstructive apnoeas were defined as a cessation of airflow lasting at least $10 \mathrm{~s}$ with associated continuous inspiratory efforts. We considered for each apnoea two segments: the preapnoeic period and the obstructive period. These segments were defined using all polygraphic data to determine as accurately as possible the beginning and the end of apnoea. The analysis of some variables has been partially described in detail elsewhere [14].

During the three unoccluded breaths preceding the onset of apnoea, inspiratory peak oesophageal pressure $(P$ oes) and the peak inspiratory flow were measured. The mean preapnoeic oesophageal pressure was defined as the average of the single measured values. Resistance at peak inspiratory flow between apnoeic events was calculated as the ratio of each oesophageal pressure to peak inspiratory flow; the values obtained for each preapnoeic breath were averaged to obtain the mean resistance at peak inspiratory flow (RPIF). As before the start of apnoea, the last unoccluded breath may show flow limitation, we also measured the $R$ PIF before the apnoea (RPIF,pre) in the second and third-last breaths preceding the apnoea, which were free from flow limitation as judged from the shape of the inspiratory flow curve.

During apnoea, the swings in oesophageal pressure during all occluded efforts were measured, and the following indices were calculated: the number of occluded respiratory cycles (cycles), the minimum of oesophageal pressure swings recorded at the start of apnoea, the maximum of the final swings $\left(P_{\text {oes, }}\right.$ max $)$ recorded at the end of apnoea, the difference between the minimum and the maximum $\left(\Delta P_{\mathrm{oes}}\right)$ of the oesophageal pressure swings, and the rate of increase in intrathoracic pressure (RPoes), defined as the ratio of $\Delta P$ oes to the duration of apnoea. We also measured the lowest $\mathrm{Sa}_{\mathrm{a}} \mathrm{O}_{2}$ value recorded after each apnoea and the value before occlusion in order to determine for each apnoea the decrease in oxygen saturation $\left(\Delta \mathrm{Sa}_{\mathrm{a}} \mathrm{O}_{2}\right)$ and its rate of decrease $\left(\mathrm{RSa}, \mathrm{O}_{2}\right)$, defined as the ratio of $\Delta \mathrm{Sa}_{\mathrm{a}} \mathrm{O}_{2}$ to the apnoea duration. As indices of respiratory drive, we considered the value of $\mathrm{R} P$ oes and the $P_{\text {oes }}$ at the first occluded breath $\left(P_{\text {oes }}, 1\right)$.

During quiet wakefulness prior to sleep onset and in supine position, we defined for each patient the awake $\mathrm{Sa}_{\mathrm{a}} \mathrm{O}_{2}$, the mean peak inspiratory flow and the mean peak inspiratory oesophageal pressure taken from the average of five consecutive breaths; their ratio was used as an awake resistance at peak inspiratory flow (RPIF,w).

Results are given as means_sem. Data for each $1 \mathrm{~h}$ period obtained from each subject were averaged, and the slope of variation of ventilatory variables for the $1 \mathrm{~h}$ sample was determined with regression analysis over time. Values of ventilatory variables at each time point were compared by repeated measure analysis of variance. Pearson's correlation and multiple regression analysis were used to determine the relationship between changes in ventilatory variables and nocturnal and diurnal parameters. Statistical significance was assumed at $\mathrm{p}$-value $<0.05$.

\section{Results}

Clinical, anthropometric and nocturnal respiratory findings of the patient group are listed in table 1.

The patients, aged $52.8 \pm 3.0$ yrs with a mean body mass index (BMI) of $30.8 \pm 1.4 \mathrm{~kg} \cdot \mathrm{m}^{-2}$, had severe OSA syndrome, with a mean AHI of 85.9 \pm 6.8 ranging 55-128 and a mean AI of $82.9 \pm 6.8$. The apnoeas were predominantly obstructive $(94 \%)$ with a small number of central apnoeas $(1 \%)$ and mixed apnoeas $(5 \%)$. A wide range of nocturnal 
Table 1. - Anthropometric, clinical and polysomnographic data of analysed patients

\begin{tabular}{|c|c|c|c|}
\hline & Mean & SEM & Range \\
\hline Age yrs & 52.9 & 3.0 & $39-66$ \\
\hline $\begin{array}{l}\text { Body mass index } \mathrm{kg} \cdot \mathrm{m}^{-2} \\
\text { FEV } 1 / \text { FVC } \%\end{array}$ & 30.8 & 1.4 & $25-36$ \\
\hline $\mathrm{Pa}_{\mathrm{a}, \mathrm{O}_{2}} \mathrm{mmHg}$ & 77.1 & 2.6 & $67-89$ \\
\hline$P \mathrm{a}, \mathrm{CO}_{2} \mathrm{mmHg}$ & 74.0 & 4.1 & 59-90 \\
\hline AHI $h^{-1}$ & 38.8 & 1.7 & $34-48$ \\
\hline Mean lowest $\mathrm{Sa}_{\mathrm{a}, \mathrm{O}_{2}} \%$ & 85.9 & 6.8 & $57-128$ \\
\hline Minimal $\mathrm{Sa}_{\mathrm{a}} \mathrm{O}_{2} \%$ & 87.0 & 1.5 & 78-93 \\
\hline Awake $\mathrm{Sa}_{\mathrm{a}} \mathrm{O}_{2} \%$ & 66.0 & 3.6 & $40-75$ \\
\hline Awake $P$ oes $\mathrm{cmH}_{2} \mathrm{O}$ & 95.8 & 0.49 & 93-98 \\
\hline Awake resistance inspiratory & 7.0 & 1.13 & $3-13$ \\
\hline flow $\mathrm{cmH}_{2} \mathrm{O} \cdot \mathrm{L}^{-1} \cdot \mathrm{S}$ & 8.1 & 1.19 & $2.9-16$ \\
\hline
\end{tabular}

FEV1: forced expiratory volume in one second; FVC: forced expiratory vital capacity; $\mathrm{Pa}, \mathrm{O}_{2}$ : oxygen tension in arterial blood; $P \mathrm{a}, \mathrm{CO}_{2}$ : carbon dioxide tension in arterial blood; $\mathrm{Sa}_{\mathrm{a}} \mathrm{O}_{2}$ : arterial oxygen saturation; $P$ oes: oesophageal pressure.

hypoxaemia severity was present with a mean lowest $\mathrm{Sa}_{\mathrm{a}} \mathrm{O}_{2}$ of $87.0 \pm 1.5 \%$ and a minimal $\mathrm{Sa}_{2} \mathrm{O}_{2}$ of $66.0 \pm 3.6 \%$.

As a group, the patients had normal blood gases, forced expiratory volume in one second (FEV1) and FEV1/forced vital capacity (FVC)\%. Two patients were hypoxaemic (arterial oxygen tension $\left(\mathrm{Pa}_{\mathrm{a}} \mathrm{O}_{2}\right) 8.6 \mathrm{kPa}(ð 65 \mathrm{mmHg})$ ) and one hypercapnic (arterial carbon dioxide tension $\left(\mathrm{Pa}, \mathrm{CO}_{2}\right)$ $6.0 \mathrm{kPa}(\mathrm{S} 45 \mathrm{mmHg}))$. None of the patients showed any restrictive or obstructive lung disease.

Nocturnal sleep structure was disrupted in all patients with a mean total sleep time of $318.6 \pm 25$ min, a mean sleep efficiency of $70.1 \pm 5.3 \%$, a mean WASO of $135.9 \pm$ $23.3 \mathrm{~min}$, and a mean number of arousals, awakenings, and sleep state changes of $220.7 \pm 40.1,221.9 \pm 37.4$ and $327 \pm 98.5$, respectively. Sleep architecture showed in all patients an absence of slow wave sleep, a reduced amount of REM sleep $(9.4 \pm 1.4 \%)$ and a greater amount of light sleep (stage 1: $21.7 \pm 4.1 \%$, stage 2: $68.9 \pm 3.5 \%$ ).

In the awake state before sleep onset, the mean oesophageal pressure was $7.0 \pm 1.1 \mathrm{cmH}_{2} \mathrm{O}$ (range: $3-13$ ), the mean $\mathrm{Sa}_{\mathrm{a}} \mathrm{O}_{2} 95.8 \pm 0.5 \%$ (range: 93-98) and the mean $R$ PIF,w $8.1 \pm 1.2 \mathrm{cmH}_{2} \mathrm{O} \cdot \mathrm{L}^{-1} \cdot \mathrm{s}$ (range: $3-16$ ).

A total of 1,752 apnoeas were analysed with a mean duration of $29.9 \pm 0.2 \mathrm{~s}$ and a mean postapnoeic $\mathrm{Sa}, \mathrm{O}_{2}$ of
$87.6 \pm 0.12 \%$. Before apnoea, the mean RPIF was $16.3 \pm 0.2$ $\mathrm{cmH}_{2} \mathrm{O} \cdot \mathrm{L}^{-1} \cdot \mathrm{s}$ and the mean preapnoeic $P$ oes $23.8 \pm 0.3 \mathrm{~cm}$ $\mathrm{H}_{2} \mathrm{O}$. At the start of the apnoea, $P_{\text {oes }}$ dropped at a minimal value of $20.1 \pm 0.2 \mathrm{cmH}_{2} \mathrm{O}$ during the first or second occluded breath; then, it progressively increased until it reached an average maximum $(P$ oes, max $)$ of $50.8 \pm 0.4 \mathrm{~cm}$ $\mathrm{H}_{2} \mathrm{O}$. The maximal $P$ oes was recorded during the last or second to last breath before breathing resumption. The difference between the minimum to the maximum $P_{\text {oes }}$ ( $\triangle P_{\text {oes }}$ ) was $30.7 \pm 0.4 \mathrm{cmH}_{2} \mathrm{O}$, the average $R P$ oes was 1.1 $\mathrm{cmH}_{2} \mathrm{O} \cdot \mathrm{s}^{-1}$ and the $\mathrm{Sa}, \mathrm{O}_{2}$ decreased by a mean value of $8.9 \pm 0.1 \%$ during the apnoea.

The polygraphic data of the analysed apnoeas during every hour of the night are given in table 2 . As the night progressed, the apnoea duration significantly increased from an initial value of 26 to a final value of $31.3 \mathrm{~s}$ $(\mathrm{F}: 2.80, \mathrm{p}=0.02)$, associated with an increase in the number of occluded breaths from 5.9 to $7.7(p=0.017)$. The overall indices of respiratory effort increased significantly during the night. The $P$ oes,max rose from a mean value of $39.5 \mathrm{in}$ the first hour to a mean value of $51.8 \mathrm{cmH}_{2} \mathrm{O}$ in the last hour $(\mathrm{F}: 2.81, \mathrm{p}=0.02)$. A concomitant rise in $\Delta P_{\mathrm{oes}}$ was noted $(\mathrm{F}: 3.47, \mathrm{p}=0.006)$ from an initial value of 21.5 to a final value of $34.9 \mathrm{cmH}_{2} \mathrm{O}$. As far as the indices of chemosensitivity are concerned, the $P_{\text {oes }}$ value at the first occluded breath did not differ during the night, whereas $\mathrm{R}$ Poes ( $\mathrm{p}=0.013$ ) rose significantly throughout the night. Despite the increase in apnoea duration, the three indices of nocturnal hypoxaemia, i.e. $\mathrm{Sa}, \mathrm{O}_{2}$ after, $\Delta \mathrm{Sa}_{\mathrm{a}} \mathrm{O}_{2}$ and $\mathrm{RSa}, \mathrm{O}_{2}$, and the index of pulmonary resistance did not undergo any consistent changes from the first to the last hour of the night.

The mean values \pm SEM of $P_{\text {oes,max }}$, apnoea duration and $\Delta P$ oes are plotted in figure 1 . The figure shows the typical trend of evolution of the three variables, Regarding apnoea length, the maximal rise was seen within the first $2 \mathrm{~h}$ of sleep; then, it reached a plateau, followed by a small decrease in the last hour of the night. A similar evolution was noted for $P_{\text {oes,max, }}$ whereas $\Delta P$ oes showed a trend in continuous rise. In contrast (figs. 2 and 3), $P$ oes, 1 and $R$ PIF exhibited a substantial increase during the first $4 \mathrm{~h}$ of sleep followed by an inverse evolution in the second part of the night.

Table 2. - Mean values of ventilatory variables for each $1 \mathrm{~h}$ period during nonrapid eye movement (NREM) sleep

\begin{tabular}{|c|c|c|c|c|c|c|c|c|}
\hline & Hour 1 & Hour 2 & Hour 3 & Hour 4 & Hour 5 & Hour 6 & Hour 7 & Slope \\
\hline NREM sleep $\min$ & 41.2 & 38.7 & 39.6 & 42.0 & 38.12 & 46.6 & 43.32 & \\
\hline REM sleep min & 0 & 2.6 & 1.9 & 2.9 & 4.5 & 6.2 & 5.7 & \\
\hline Number of recorded apnoeas & 381 & 474 & 509 & 421 & 485 & 532 & 441 & \\
\hline Number of analysed apnoeas & 304 & 271 & 345 & 234 & 247 & 170 & 154 & \\
\hline Duration $\mathrm{s}$ & 25.7 & 29.7 & 28.4 & 29.9 & 31.1 & 31.2 & 31.3 & 0.80 \\
\hline $\mathrm{S}_{\mathrm{a}, \mathrm{O}_{2}}$ after $\%$ & 89.2 & 86.6 & 87.6 & 87.6 & 87.0 & 87.2 & 88.1 & -0.10 \\
\hline$\Delta S_{\mathrm{a}, \mathrm{O}_{2}} \%$ & -7.5 & -9.9 & -8.8 & -9.1 & -9.7 & -9.6 & -8.4 & -0.10 \\
\hline $\mathrm{RSa}, \mathrm{O}_{2} \%$ & -0.30 & -0.40 & -0.31 & -0.31 & -0.30 & -0.30 & -0.26 & -0.01 \\
\hline$R$ PIF $\mathrm{cmH}_{2} \mathrm{O} \cdot \mathrm{L}^{-1} \cdot \mathrm{s}$ & 15.5 & 16.4 & 17.3 & 17.6 & 16.5 & 15.2 & 15.0 & -0.16 \\
\hline$R$ PIF,pre $\mathrm{cmH}_{2} \mathrm{O} \cdot \mathrm{L}^{-1} \cdot \mathrm{s}$ & 12.2 & 12.7 & 13.7 & 13.7 & 13.9 & 12.1 & 12.5 & 0.00 \\
\hline$P_{\text {oes }, 1} \mathrm{cmH}_{2} \mathrm{O}$ & 19.7 & 24.0 & 22.2 & 22.3 & 24.0 & 23.7 & 18.7 & -0.07 \\
\hline$P_{\text {oes, } \max } \mathrm{cmH}_{2} \mathrm{O}$ & 39.5 & 51.2 & 50.6 & 52.9 & 55.4 & 57.1 & 51.8 & 1.91 \\
\hline$\Delta P_{\text {oes }} \mathrm{cmH}_{2} \mathrm{O}$ & 21.5 & 29.6 & 30.3 & 32.7 & 33.8 & 36.7 & 34.9 & 2.07 \\
\hline $\mathrm{R}$ Poes $\mathrm{cmH}_{2} \mathrm{O} \cdot \mathrm{s}^{-1}$ & 0.85 & 1.01 & 1.10 & 1.11 & 1.12 & 1.19 & 1.17 & 0.05 \\
\hline
\end{tabular}

$\mathrm{Sa}_{\mathrm{a}} \mathrm{O}_{2}$ : arterial oxygen saturation; $\Delta \mathrm{Sa}_{\mathrm{a}} \mathrm{O}_{2}$ : decrease in arterial oxygen saturation; $\mathrm{RSa}, \mathrm{O}_{2}$ : rate of decrease in arterial oxygen saturation; $R$ PIF: pulmonary resistance at peak inspiratory flow; $R$ PIF,pre: $R$ PIF during the second and third-last breaths preceding the apnoea;

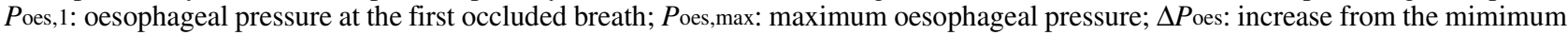
to the maximum oesophageal pressure; $R$ Poes: rate of increase in oesophageal pressure. 

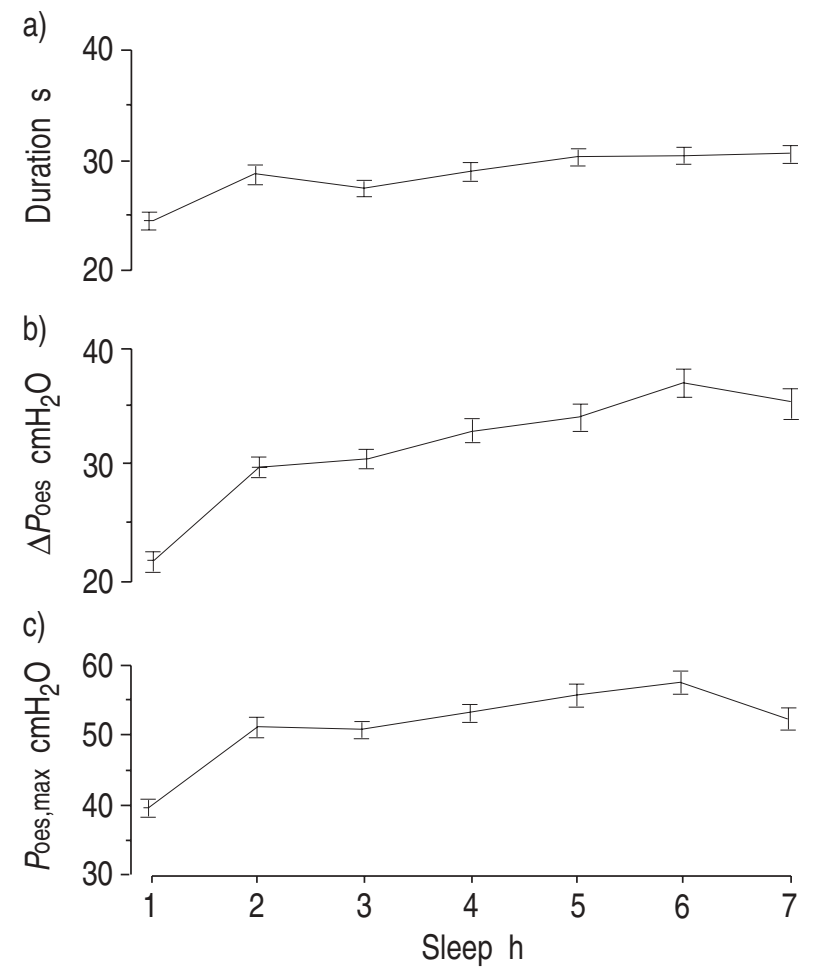

Fig. 1. - The mean values \pm SEM of apnoea length and indices of respiratory effort for $7 \mathrm{~h}$ of sleep. We noted in the group of patients a trend in progressive increase in apnoea duration (a) associated with a significant rise in increase from the minimum to the maximum oesophageal pressure $\left(\Delta P_{\text {oes }} ; \mathrm{b}\right)$ and maximal end-apnoeic oesophageal pressure $\left(P_{\mathrm{oos}, \max }\right.$; c). The maximal rise was present within the first hours of sleep; then a plateau was reached followed by a decrease in the last hour of the night.

A Pearson's correlation analysis including the slope of increase in inspiratory effort and indices of sleep fragmentation and diurnal and nocturnal variables showed that none of the variables considered was correlated with the changes in Poes,max during the night. A significant and positive correlation was found between the slope of increase in apnoea length $(\mathrm{r}=0.67, \mathrm{p}=0.05)$ and $P$ oes,max $(\mathrm{r}=0.66, \mathrm{p}=0.05)$ and the AHI.

\section{Discussion}

The results of this study have demonstrated that throughout the night, the recurrence of apnoeas results in altered responses to airway occlusion, including prolongation of the apnoea duration and increased peak inspiratory effort, irrespective of changes in hypoxaemia, respiratory drive responsiveness and pulmonary resistance. Using the $P_{\text {oes, }}$ max as an index of arousal threshold, we also demonstrated that repetition of apnoeic stimulus results in similar changes in the arousal threshold to occlusion. This latter finding suggests that the overnight rise in apnoea duration can be attributed predominantly to the associated change in arousal threshold rather than to other factors, such as nocturnal hypoxaemia or changes in upper airway collapsibility and chemosensitivity.

Some concerns about the methodology that we employed should be discussed before the analysis of our results. The measurements of respiratory variables we made may be affected by the distribution of sleep periods during the night as well as by the degree of sleep alteration. To mini-

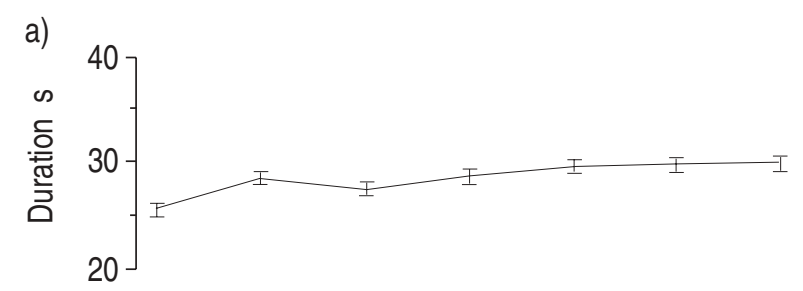

b)
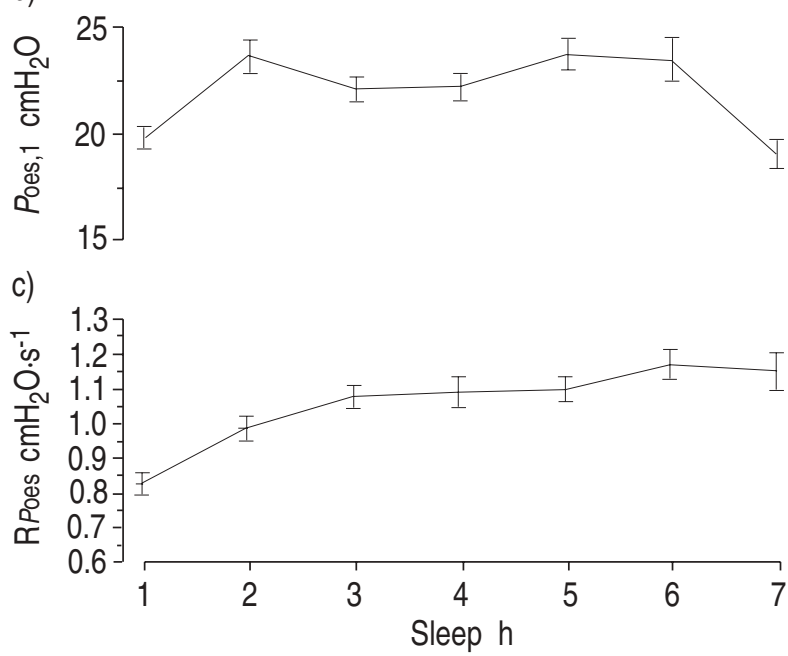

Fig. 2. - The evolution during the night of apnoea length (a) and two indices of chemosensitivity, the oesophageal pressure at the first occluded breath $\left(P_{\text {oes }}, 1 ; \mathrm{b}\right)$ and the rate of increase in oesophageal pressure $(\mathrm{R} P$ oes; $\mathrm{c})$. For RPoes, a gradual and continuous increase with a maximal rise during the first $4 \mathrm{~h}$ of sleep was noted. The $P_{\mathrm{oes}}, 1$ showed a tendency to decline gradually in the second part of the night.

mize the effect of prolonged awakenings, we selected patients for whom repetitive apnoeas were present for the entire night with wakefulness periods lasting $<30 \mathrm{~min}$. Moreover, since the rise in apnoea duration over the night might merely reflect the greater amount of REM sleep in the second part of the night, we limited the analysis to NREM sleep. An upward trend in apnoea length and in $P_{\text {oes,max }}$ can still be observed, suggesting that the results cannot be attributed to the effects of REM sleep in itself. Moreover, our laboratory [14] has previously demonstrated that all indices of respiratory effort are lower in REM sleep. Taken together, these data led us to exclude the interference of REM sleep on nocturnal respiratory effort changes. Secondly, we have used the resistance at peak inspiratory flow as an index of upper airway resistance, which does not address directly upper airway collapsibility and dimension. It is known that upper airway resistance constitutes most of RPIF during NREM sleep and changes in RPIF are correlated with changes in upper airway resistance [15]. The use of this index may be complicated by the fact that the airway resistance can vary within a breath because of the variable negative pressure applied during inspiration to the collapsible airway. To avoid the confounding effect of flow limitation occurring frequently at the last breath before the onset of apnoea, we measured the peak inspiratory resistance at the second and third breath before the apnea in which the relationship between flow rate and negative pressure is often linear [15]. Even though we considered values without flow limitation, the 
a)

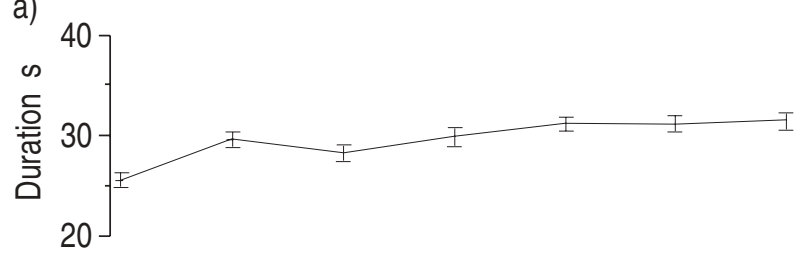

b)

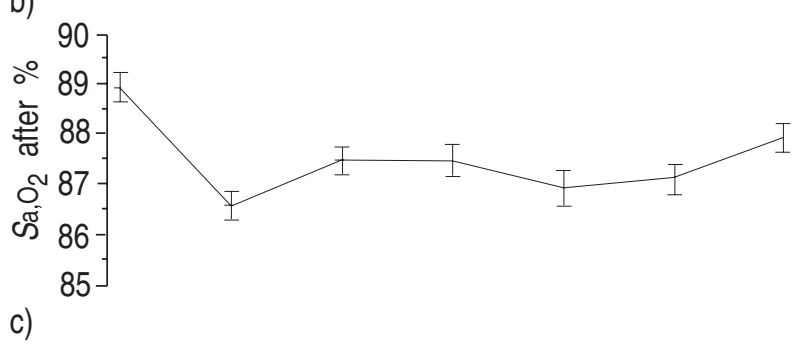

c)

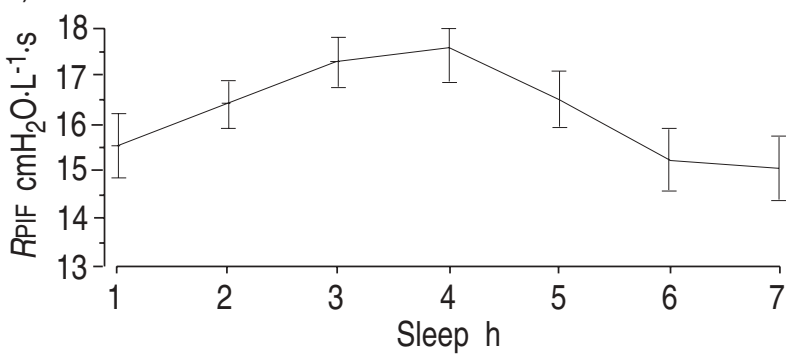

Fig. 3. - The figure shows the evolution during the $7 \mathrm{~h}$ of sleep for apnoea duration (a), low arterial oxygen saturation $\left(\mathrm{Sa}_{\mathrm{a}} \mathrm{O}_{2} ; \mathrm{b}\right)$ and inspiratory peak resistance $(R \mathrm{PIF} ; \mathrm{c})$ before apnoea. For the preapnoeic resistance, the trend was in the opposite direction compared with the evolution of apnoea duration with an increase during the first $4 \mathrm{~h}$ of the night and a decrease in the second part of the night. After a maximal fall occurring during the second hour of the night, no significant changes in nocturnal hypoxaemia were found during the remaining hours.

resistance at peak inspiratory flow did not appear to increase during the night. Finally, one might also query whether the $P$ oes,max is an appropriate index of arousal threshold in patients with sleep-related breathing disorders. Despite the limits of this definition, several experimental and clinical observations $[2-4,16]$ indicate that the most important factor in apnoea termination and in arousal response is the maximum level of oesophageal pressure recorded at the end of apnoea, and consequently, the change in $P_{\text {oes,max }}$ during the night may reflect variation in arousability.

The new finding of our study is that there is a progressive increase in apnoea duration present across the night associated with a significant rise in $P_{\text {oes, }}$ max (i.e. the index of arousal response to occlusion), and that occurs irrespective of changes in nocturnal hypoxaemia, in respiratory drive response and in preapnoeic resistance. These results are in keeping with the clinical observation that in the OSA syndrome, a progressive increase in apnoea duration occurs across the night $[17,18]$ and with the data of MONTSERRAT et al. [10] showing a concomitant increase in $P$ oes,max and apnoea duration at the end of the night. However, these studies were limited to an analysis at the start and at the end of the night for a limited number of apnoeas. Using a 1-h analysis, we were able to demonstrate that the increase in Poes,max and apnoea duration was greater during the first $2 \mathrm{~h}$ of the night, followed by a plateau in the second half of the night. This later finding may suggest that the changes in arousal response to upper air- way occlusion can be attributed either to overnight alterations in the arousal threshold of the central nervous system or as being secondary to sleep fragmentation and recurrent apnoeas.

The potential mechanisms underlying the nocturnal changes in apnoea duration and in the index of arousal threshold are still not clear. We know that the arousal at the end of an obstructive apnoea may be induced by afferent stimuli arising from chemoreceptors, upper airway mechanoreceptors as well as increased ventilatory effort. Thus, several mechanisms may be implicated in the apnoea lengthening and in the increase in Poes,max during the night: changes in central chemosensitivity and in upper airway collapsibility, a dysfunction of peripheral mechanoreceptors, or altered arousal mechanisms.

Though upper airway resistance is higher in OSA patients when awake, it is well established that sleep per se $[19,20]$ or sleep fragmentation [21] has an impact on the upper airway collapsibility. If the rise in respiratory effort found in our OSA patients is influenced by changes in preapnoeic collapsibility, we could expect that a progressive increase in RPIF over the night would be present and shorter apnoeas might be recorded at the end of the night since a shorter time is necessary to obtain the maximal value of $P_{\text {oes }}$ causing arousal [22]. Peak inspiratory resistance did not change across the night and thus proved against the possibility that increased upper airway resistance contributed to the $P_{\text {oes, }}$ max rise.

It is well known that the maximal intrathoracic pressure generated during occlusion is a measure of the respiratory drive activated by chemical and mechanical inputs [23]. As indices of respiratory drive influence, we consider the first value of oesophageal pressure at the start of occlusion [23] and the rate of increase of respiratory effort during the apnoea $[4,11]$. When these variables were analysed for each hour of the night, $P_{\text {oes }}, 1$ did not show significant changes whereas a significant increase in $\mathrm{R}$ Poes was found. This suggests that the only effect of respiratory drive during sleep is to influence the rate at which inspiratory effort rises to provoke arousal. However, the possibility that the arousal responsiveness to occlusion depends on the alteration of hypercapnic respiratory drive response cannot be ruled out $[5,6,23]$ since end-tidal $\mathrm{CO}_{2}$ was not measured in this study.

Increased apnoea duration and $P_{\text {oes,max }}$ during the night may also reflect a blunted arousal response as an adaptation to repetition of apnoeic stimuli. The reduced arousability through the night could be related either to a progressive decline in peripheral sensitivity or to a decrease in central responsiveness to peripheral stimuli. The influence of a nocturnal decline in peripheral sensitivity is suggested by the study of CALA et al. [24], showing that anaesthesia of the upper airway resulted in an increased respiratory effort and apnoea length at the start of sleep without any effect in the second part of the night. This observation suggests that afferents arising from the upper airway mechanoreceptors may exert an inhibitory influence directly on the respiratory drive at the start of sleep.

Although our results do not allow us to establish a casual relationship between sleep fragmentation and change in $P$ oes,max, an attractive theory is the lowering of arousal threshold in OSA induced by sleep fragmentation and recurrence of apnoeas. On the basis of the relationship between changes in $P_{\text {oes,max }} \Delta \mathrm{P}_{\mathrm{Pes}}$ and AHI, we can suppose 
that sleep deprivation and sleep fragmentation have an effect on arousal responsiveness to respiratory stimuli either throughout the night or in the natural course of the disease [11]. An increased amount of time to reach arousal and an increased tendency to upper airway collapse have been reported after short-term sleep fragmentation in animals [25, 26], in normals [22] and in OSA patients [8], ex-plaining the deleterious effect of benzodiazepines [27, 28] and alcohol [29]. The detrimental effects of chronic sleep fragmentation on arousal response are evident from the re-sults of BRooKs et al. [9] who studied four dogs in an OSA and sleep fragmentation protocol, and did not find any differences in apnoea length, time of arousal and peak inspiratory pressure in both conditions. In our subjects, however, the number of arousals and awakenings does not influence the nocturnal trend in arousal threshold. This finding may be explained by the difficulty to assess sleep fragmentation visually. Since resolution of apnoea is not necessarily associated with a cortical arousal or a "microarousal" [30], more sophisticated EEG analysis may be useful to assess the role of sleep fragmentation on the arousal response to occlusion.

Using a 1-h analysis we tried to obtain a dynamic interpretation of the changes in arousal threshold in patients with OSAs. The scattergram of the changes in apnoea length and in $P_{\text {oes,max }}$ against the hour of the night revealed a peculiar trend of evolution. Interestingly, the rise in the two variables was not linear, with a greatest rise recorded in the first hours of the night followed by a plateau after the third hour and a tendency to decrease in the last part of the night. One possible explanation of these results is the methodological bias related to the analysis of selected apnoeas during NREM sleep. Since, as shown in table 2 , no changes in the recorded apnoeas were noted across the night, the trend in decrease or in plateau found in the second part of the night may be related to the small number of apnoeas analysed, which in turn is related to the greater amount of REM sleep recorded in the last hours of the night. A second and more interesting possibility is that arousability may be modulated by circadian factors and, as a result, change through the course of the night. This seems intuitively logical when we consider the relationship between the circadian rhythm of the sleep promoting system and the change in arousability. Using an ultra-short multiple naps protocol LAVIE and SCHERSON [31] have shown that a rapid rise occurs in sleep propensity, beginning at about 20:00 $\mathrm{h}$ followed by a broad plateau from midnight to 06:00 h. Considering this model of circadian sleep propensity, the mechanical response necessary to provoke arousal may be proportionally greater at the start of sleep when the sleepiness level is higher, compared with that at higher levels of alertness. Although this hypothesis is purely speculative at the present time, an interference of circadian rhythm is provided by a recent report [32], demonstrating that $P$ oes,max parallels the decline of delta activity in OSA patients, reflecting the physiological decrease in sleep pressure during the night.

In summary, we have found in patients with obstructive sleep apnoea a nocturnal rise of respiratory effort and apnoea duration occurring without any changes in oxygen desaturation, in chemosensitivity and in preapnoeic upper airway resistance. The frequency of sleep-related breathing disorders appears to contribute to the increase in apnoea length and in maximal oesophageal pressure supporting the hypothesis of a resetting of arousal threshold in obstructive sleep apnoea syndrome. Since a time-dependent evolution in maximal oesophageal pressure was found, further investigations should be directed at establish whether sleep fragmentation or circadian influence is implicated in arousal threshold change in obstructive sleep apnoea syndrome.

Acknowledgements: The authors would like to thank C. Reppel, E. Georges, M. Favier and M.P. Bilger for monitoring the sleep recordings and to C. Bonigen, B. Kowalski, C. Mansouri, and A. Peter for scoring the sleep stages.

\section{References}

1. Onal E, Leech JA, Lopata M. Dynamics of respiratory drive and pressure during NREM sleep in patients with occlusive apnea. J Appl Physiol 1985; 48: 432-437.

2. Gleeson K, Zwillich CW, White DR. The influence of increasing ventilatory effort on arousal from sleep. Am Rev Respir Dis 1990; 142: 295-300.

3. Vincken W, Guilleminault C, Silvestri L, Cosio M, Grassino A. Inspiratory muscle activity as a trigger causing the airways to open in obstructive sleep apnea. Am Rev Respir Dis 1987; 135: 372-377.

4. Kimoff RJ, Cheong TH, Olha AE, et al. Mechanisms of apnea termination in obstructive sleep apnea. Role of chemoreceptor and mechanoreceptor stimuli. Am Rev Respir Dis 1994; 149: 707-714.

5. Kimoff RJ, Kozar LF, Yasuma F, Bradley TD, Phillipson EA. Effect of inspiratory muscle unloading on arousal responses to $\mathrm{CO}_{2}$ and hypoxia in sleeping dogs. $J$ Appl Physiol 1993; 74: 1325-1336.

6. Kim WS, Champagne VL, Gottfried SB, Kimoff RJ. Effect of inspiratory pressure support on the arousal response to $\mathrm{CO}_{2}$ in sleeping dogs. J Appl Physiol 1995; 79: 1419-1425.

7. Yasuma FL, Kozar LF, Kimoff RJ, Bradley TD, Phillipson EA. Interaction of chemical and mechanical respiratory stimuli in the arousal response to hypoxia in sleeping dogs. Am Rev Respir Dis 1991; 143: 1274-1277.

8. Persson HE, Svanborg E. Sleep deprivation worsens obstructive sleep apnea. Comparison between diurnal and nocturnal polysomnography. Chest 1996; 109: 645-650.

9. Brooks D, Horner RL, Kimoff RJ, Kozar LF, RenderTeixeira CL, Phillipson EA. Effect of obstructive sleep apnea versus sleep fragmentation on response to airway occlusion. Am J Respir Crit Care Med 1997; 155: 16091617.

10. Montserrat JM, Kosmas EN, Cosio MG, Kimoff RJ. Mechanisms of apnea lengthening across the night in obstructive sleep apnea. Am J Respir Crit Care Med 1996; 154: 993-995.

11. Berry RB, Kouchi KG, Der DE, Dickel MJ, Light RW. Sleep apnea impairs the arousal response to airway occlusion. Chest 1996; 109: 1490-1496.

12. Guilleminault C, Roseland M. The arousal threshold: sleep deprivation, sleep fragmentation and obstructive sleep apnea syndrome. Bull Eur Physiopathol Respir 1981; 17: 341-349.

13. Rechtschaffen A, Kales A. A manual of standardized terminology, technique and scoring system for sleep stages of human sleep. Brain Information Institute, UCLA, Los Angeles Brain Information Service, 1968.

14. Krieger J, Sforza E, Boudewijns A, Zamagni M, Petiau C. Respiratory effort during obstructive sleep apnea. Role of age and sleep state. Chest 1997; 112: 875-884. 
15. Begle RL, Badr S, Skatrud JB, Dempsey JA. Effect of lung inflation on pulmonary resistance during NREM sleep. Am Rev Respir Dis 1990; 141: 854-860.

16. Berry RB, Gleeson K. Respiratory arousal from sleep: mechanisms and significance. Sleep 1997; 20: 654-675.

17. Charbonneau M, Marin JM, Olha A, Kimoff J, Levy RD, Cosio MG. Changes in obstructive sleep apnea characteristics through the night. Chest 1994; 106: 1695-1701.

18. Lavie P, Halperin E, Zomer J, Alroy G. Across-night lengthening of sleep apneic episodes. Sleep 1981; 4: 279282.

19. Wiegand L, Zwillich C, White D. Sleep and the ventilatory response to resistive loading in normal man. $J$ Appl Physiol 1988; 64: 1186-1195.

20. Anch AM, Remmers JE, Bunce H III. Supraglottic airway resistance in normal subjects and patients with occlusive apnea. J Appl Physiol 1982; 55: 1158-1163.

21. Series F, Roy N, Marc I. Effects of sleep deprivation and sleep fragmentation on upper airway collapsibility in normal subjects. Am Rev Respir Dis 1994; 150: 481-485.

22. Mayer P, Champagne V, Kimoff RJ. Effect of prior flowresistive loading on the arousal response to airway occlusion. Am J Respir Crit Care Med 1995; 151: A154.

23. Berry RB, Mahutte CK, Light RW. Effect of hypercapnia on the arousal response to airway occlusion during sleep in normal subjects. J Appl Physiol 1993; 74: 22692275.

24. Cala SJ, Sliwinski, P, Cosio, MG, Kimoff RJ. Effect of topical upper airway anesthesia on apnea duration thro- ugh the night in obstructive sleep apnea. $J$ Appl Physiol 1996; 81: 2618-2626.

25. Fewell JE. The effects of short-term fragmentation produced by intense auditory stimuli on the arousal response to upper airway obstruction in lambs. J Dev Physiol 1987; 9: 409-417.

26. Bowes G, Woolf GM, Sullivan CE, Phillipson EA. Effect of sleep fragmentation on ventilatory and arousal responses of sleeping dogs to respiratory stimuli. Am Rev Respir Dis 1980; 122: 899-908.

27. Schneider H, Grote L, Peter JH, Cassel W, Guilleminault C. The effect of triazolam and flunitrazepam - two benzodiazepines with different half-lives - on breathing during sleep. Chest 1996; 109: 909-915.

28. Berry RB, Kouchi K, Bower J, Prosise G, Light RW. Triazolam in patients with obstructive sleep apnea. Am $J$ Respir Crit Care Med 1995; 151: 450-454.

29. Berry RB, Bonnet MH, Light RW. Effect of ethanol on the arousal response to airway occlusion during sleep in normal subjects. Am Rev Respir Dis 1992; 145: 445-452.

30. Martin SE, Engleman HM, Kingshott RN, Douglas NJ. Microarousals in patients with sleep apnoea/hypopnoea syndrome. J Sleep Res 1997; 6: 276-280.

31. Lavie P, Scherson A. Ultrashort sleep-waking schedule. I. Evidence of ultradian rhythmicity in sleep ability. Electroencephalogr Clin Neurophysiol 1981; 52: 163-174.

32. Berry RB, Khoo MCK, Asyali MH, McNellis MI. Cyclic variation in the arousal threshold to airway occlusion in sleep apnea. Sleep Res 1997; 26: 328. 